\title{
Philosophiques
}

\section{Le désir de dire}

\section{Patrick Drevet}

Volume 21, numéro 2, automne 1994

Les femmes et la société nouvelle

URI : https://id.erudit.org/iderudit/027293ar

DOI : https://doi.org/10.7202/027293ar

Aller au sommaire du numéro

Éditeur(s)

Société de philosophie du Québec

ISSN

0316-2923 (imprimé)

1492-1391 (numérique)

Découvrir la revue

Citer ce document

Drevet, P. (1994). Le désir de dire. Philosophiques, 21(2), 549-561.

https://doi.org/10.7202/027293ar d'utilisation que vous pouvez consulter en ligne.

https://apropos.erudit.org/fr/usagers/politique-dutilisation/ 


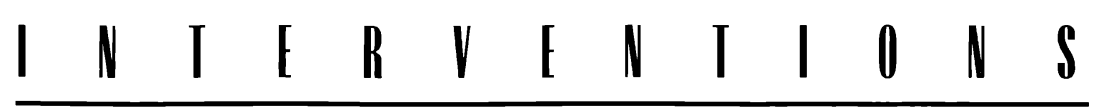

\section{Le désir de dire}

\author{
par \\ Patrick Drevet*
}

Il n'est guère de jugement littéraire qui ne s'exprime sur fond d'absolu. Nous jugeons de la qualité d'une œuvre sur la capacité qu'elle nous paraît avoir de traverser les siècles. C'est même l'argument ultime auquel nous recourons pour imposer notre avis, par un réflexe identique à celui que nous avions sur les cours du lycée et qui pouvait nous conduire jusqu'a échanger des coups. Il présente l'avantage de clore toute discussion et permet d'être péremptoire sans ridicule puisqu'il ne saurait être question pour le futur de venir à l'avance nous contredire. Corrélativement, il n'est pas sans reposer sur la conviction sousjacente de notre propre éternité dans la mesure où il suppose que nous aurons toujours la possibilité de le vérifier : c'est prendre le point de vue des anges, nous transporter dans l'au-delà, être assuré de cette part immortelle en nous qui seule peut disposer d'une aussi confondante prescience.

Nombre d'auteurs eux-mêmes ne se placent pas dans une autre perspective. La concentration exigée par la création littéraire demande un retrait ou conduit à un état second qui ressortissent peut-être, en effet, à l'audelà, ce qui a pu pousser Genet à prétendre qu'il écrivait pour les morts, mais il

- NDLR : Romancier et essayiste, Patrick Drevet, jeune auteur dans la quarantaine, a déjà publié plus d'une dizaine d'ouvrages aux Editions Gallimard, puis chez Belfond. Parmi ses derniers livres parus, citons Le rire de Mandrin, Paris, Belfond, 1993 (Prix annuel des Libraires), Dieux obscurs, Paris, Belfond, 1994.

Nous avons l'honneur de publier ici une réflexion inédite de Drevet sur son art, et, plus particulièrement, sur les rapports complexes entre l'écriture, l'expression et la littérature. Ce texte fait partie d'un ensemble de trois essais dans la même veine esthétique. On trouvera dans la prochaine livraison de Philosophiques (avril 1995) les deux autres textes, l'abondance des matières dans ce numéro nous ayant obligé à reporter leur parution. 
semble aussi, souvent, que l'état d'immortels que ces auteurs endossent déjà supplée un peu trop à l'inspiration, et l'on aurait envie de leur demander moins de l'une et plus de l'autre, ils en seraient certainement plus vrais. A l'inverse, des auteurs boudés par le succès se consolent, ou le sont, par l'assurance de la valeur « plus éternelle que l'airain » de leur œuvre. Il n'est rien qui stimule davantage, encore que ce chantage à l'immortalité n'empêche pas d'être spolié de la reconnaissance qu'on mériterait et ne permette pas de vivre des émoluments mérités par, ce qui jusqu'a la mort ne demeurera qu'une conviction intime ou, au mieux, confidentielle.

Cette référence à l'immortalité est dans les racines de l'art, dans les fondements mêmes de la conscience, dans le ressort qui pousse tout homme à créer. Elle n'est donc pas en soi la base d'un jugement très rigoureux concernant la valeur littéraire mais ne laisse pas d'entraîner ces questions corollaires sur la nature de ce que l'on entend par « littérature »: pourquoi les écrits ne subsistent-ils pas tous ? Pourquoi, parmi tout ce qui a été publié, tout n'est-il pas digne d'être retenu ? Pourquoi, parmi tout ce que nos contemporains écrivent, tout n'est-il pas publiable? Pourquoi tout écrit ne se justifie-t-il pas? Quel est le secret du talent? Qu'est-ce qui fait qu'un manuscrit est une œuvre et qu'un autre ne l'est pas?

Il n'est sans doute pas possible de répondre à ces questions car les réponses seraient connues depuis longtemps et il suffirait d'en tenir compte pour écrire, ou ne pas écrire. Or, si tant est qu'on puisse parler de crise en ce qui concerne la lecture, il ne semble pas qu'on puisse en dire autant en ce qui concerne l'écriture, à en juger du moins par la quantité toujours croissante de manuscrits soumis aux maisons d'édition. Faut-il en conclure que notre civilisation tend à ce que tout le monde aspire à dire et à être entendu plus qu'à lire et à écouter ? On pourrait le croire à l'expérience de bien des « échanges » avec nos contemporains, mais il convient de remarquer que c'est là un trait constitutif de l'être humain, et il n'est pas le propre d'une époque. $N$ 'y faudrait-il pas voir, même, l'origine, sinon de la littérature, du moins du langage? Nous naissons avec un irrépressible désir de dire, avant même que nous ayons quelque objet que ce soit à exprimer. Le seul fait de vivre est une violence à manifester sans cesse. L'énergie qui anime nos cellules est toujours à la recherche d'une issue par où se communiquer. Tout ne commence-t-il pas par un cri et ne se poursuit-il dans la mesure seulement où nous savons peu ou prou nous faire entendre?

Si tout manuscrit n'est pas appelé à s'imposer comme une œuvre, il est issu de cet impératif auquel nous sommes soumis et qui, à ce niveau, ne privilégie ni ne rabaisse personne, fait chacun aussi assujetti et aussi doué que quiconque. S'il n'est pas parvenu à dresser une forme qui se tienne ou qui convainque, à proposer une évidence qui subjugue, il reste dans ses prémisses 
cet élan qui, en soi, est tout aussi significatif que celui à l'origine des chefs d'œuvre devenus universels et emblématiques. Aussi bien tout écrit mérite-til à tout le moins l'attention. Et quand on prend en compte le temps passé à sa confection, l'énergie qu'il a fallu mobiliser pour l'élaborer, la somme de travail qui, de sa rédaction à sa présentation dactylographiée, a dû être fournie pour l'obtenir, les espoirs qu'il représente, l'idéal vers quoi il manifeste son auteur tendu, tout manuscrit mérite même le respect. S'il n'est pas l'expression d'une souffrance apte à lui conférer de la dignité, ce dont on pourrait au moins le saluer, il suppose, quoi qu'il en soit, et quel qu'il soit, un rêve de salut dont personne n'est exempt, il relève d'une aspiration que nous partageons tous, avec une sorte de candeur qui est peut-être ce que nous avons de meilleur et en tout cas de pathétique.

Sans doute l'écriture n'est-elle pas la seule médiation à même de répondre au besoin d'exprimer qui est notre fatalité, mais les autres disciplines artistiques non plus. Il en est pour le satisfaire dans le sport, d'autres dans les relations sociales, d'autres dans les études, d'autres encore dans le crime, dans la course à l'argent ou dans la pêche aux voix, d'autres dans l'imaginaire de l'imaginaire qu'est la religion. Du reste, l'écriture est une des dernières techniques que les hommes se soient donnés. Ils s'en sont passés durant des millénaires et certains aspects de leur évolution actuelle tendraient à suggérer non seulement qu'ils peuvent tout aussi aisément s'en passer mais s'organisent même en vue de s'en débarrasser tout à fait. Or, quel que soit le domaine dans lequel on ait choisi où trouver à s'exprimer, il ne semble pas qu'aucun puisse aussi idéalement permettre de le faire que l'écriture.

C'est que, tout comme l'art, l'écriture offre la possibilité de subsister audelà de la mort, même sous une forme non littéraire et réduit seulement à un nom. Plus élaborée, elle peut en outre assurer à l'individu de traverser les siècles non plus sous l'aspect d'une simple trace mais en ce qui lui semble constituer l'essentiel de son être, avec son caractère et son histoire, ou ce qui en est pour lui l'image non pas idéalisée mais nettoyée des déformations dues aux ratées de l'existence. Il en va encore, dans l'envie lancinante de se projeter dans un livre, d'un pouvoir spécifique de l'écrit, car si tout être humain est animé du désir de s'exprimer, il lui reste toujours, après qu'il l'a fait d'une manière ou d'une autre, et même par la parole ou par l'écriture, quelque chose à dire, dont il ne connaît pas distinctement l'objet mais qu'il ne pourrait compter formuler autrement que par écrit. Il n'est pas de maître en tel ou tel domaine de l'activité des hommes que ne titille l'écriture. Les personnalités politiques en fournissent de nombreux et quelquefois fameux exemples, mais les sportifs aussi, et les médecins, et les peintres, les cinéastes, les musiciens, les comédiens, les chanteurs, les danseurs...

Force est d'admettre que l'écriture apparaît de façon assez paradoxale comme la possibilité d'avoir accès à ce que nous ne pouvons pas dire, à ce qui reste en travers de notre gorge, à ce qui nous encombre le cœur, à ce qui menace de nous étouffer. Qui sait si les maladies psychiques, les crises de démence, la 
folie n'affectent pas des personnes qui n'ont pu ou n'ont su trouver un échappatoire dans l'écriture? Les séances d'analyse prônées par Freud ne manquent pas en tout cas de présenter des analogies avec l'écriture, l'analyste s'offrant, par sa posture consciencieuse de scribe, attentif, silencieux, comme un substitut de l'écrivain que le patient n'a pas trouvé à devenir pour lui-même, et se chargeant, comme l'écrivain encore puisant la matière de ses ouvrages dans ses rêves, dans les travestissements de sa conscience et le chaos de sa mémoire, d'aider à se saisir de l'extrémité du fil perdu afin d'en dérouler l'écheveau. Il n'y a pas là l'essence de la littérature, qui est encore autre chose, mais c'est bien sous l'aspect de ce fil d'Ariane que nous l'envisageons communément, que ce soit pour atteindre sans nous perdre les monstres inconcevables qui nous tourmentent ou pour sortir du labyrinthe de nos errances quand nous en venons, sinon à désirer un sens à notre vie, du moins à aspirer en choisir la direction.

Il y a de fortes chances pour que la littérature - je ne dis pas l'écriture soit née de ce désir là qui, par l'invention des mythes, des contes, des récits, s'est investie du pouvoir de maîtriser les pulsions, de conjurer les terreurs, d'expliquer le monde, de divulguer ses arcanes et les forces qui présidèrent à sa création et se sont enfouies, toujours influentes, sous les apparences. Il est clair que ce même désir continue de donner à la littérature son histoire. Le souffle dont il l'anime s'est diffracté en plusieurs courants qui, au gré de la nature et des matières qu'ils rencontraient, ont engendré des formes diverses, lesquelles évoluent et évolueront encore.

Quelles que soient ces formes, la littérature serait sans doute mieux comprise si l'on en revenait à ce désir qui l'initie. C'est dans la force et la qualité de ce désir que l'on peut juger de l'authenticité, de la nécessité, de la valeur d'une œuvre, et trouver les raisons qui justifient la pérennité des unes, l'oubli où sombrent les autres, et qui sélectionnent impitoyablement dans la production incommensurable des écrits. Il y aurait là de quoi réformer la critique classificatrice, scolaire et mortifère à laquelle la littérature est soumise. Contaminée par les méthodes scientifiques, elle ne sait guère appréhender les œuvres qu'en les disséquant, ce qui suppose qu'elle les tue ou les torture jusqu'à la mort. Le biologiste qui étudie une fleur commence par l'arracher, puis il l'effeuille, la découpe, la broie, en fait un jus méconnaissable pour la passer au microscope. Il n'arrive pas autre chose aux œuvres littéraires qu'on n'en finit pas de passer à toutes les grilles possibles quand il suffirait sans doute, pour les comprendre, les pénétrer, les apprécier, d'avoir toujours par devers soi, en les lisant, la question suffisamment redoutable et sélective : qu'as-tu fait du désir de dire de ton auteur?

\section{III.}

L'origine de la littérature n'est pas liée à celle de l'écriture mais ce n'est bien qu'avec cette dernière qu'elle a acquis son statut et commence, sans doute, de découvrir sa nature, tout en suscitant l'idée de ce monde enviable dont 
l'accès serait à même de mettre définitivement à l'abri des contingences. Mais si littérature et écriture sont à ce point mêlées qu'elles en viennent à se confondre et qu'il est difficile de penser l'une sans penser l'autre, et même de ne pas les prendre l'une pour l'autre, qu'est-ce qui, précisément, suscite le plus la convoitise quand on les évoque, de l'état où l'on imagine que l'on est plongé lorsqu'on écrit ou de la plénitude qu'on suppose atteinte par l'achèvement d'un ouvrage susceptible de porter son auteur au-delà des limites de sa vie? Autrement dit, envie-t-on les facultés dont témoigne la maîtrise de l'écriture ou le prestige que la littérature confère à ses officiants, devenus d'autant plus fabuleux que, pour certains d'entre eux, la littérature n'avait d'autre fonction que d'assurer leur légende?

A l'échelle de l'histoire humaine, la consécration d'un individu par les Lettres est récente. Longtemps le titre de l'œuvre a primé sur son créateur, du moins hors du cercle étroit des clercs, et les œuvres étaient plus ou moins collectives, elles s'enrichissaient au gré de l'inspiration et de la fantaisie des conteurs qui les répandaient, des continuateurs qui les reprenaient, des copistes qui les fixaient. Longtemps encore après l'invention de l'écriture, seule primait la littérature chargée de transmettre mythes, fables, panégyriques, chansons à danser, poèmes pour égayer les soirées, pour fleurir des instants de la vie et quelquefois pour faire réfléchir. Transmise oralement, elle apparaissait non comme un produit de l'écriture mais comme une parole si virtuose, si pénétrante et troublante, qu'elle ne pouvait être que soufflée par les dieux ou tombée de leur bouche même. Ou bien c'était un arrangement musical de la langue, une jonglerie verbale, un carnaval de mots, et les formes rigides de la versification figeaient des blocs de texte que le rythme et les rimes aidaient à garder en tête.

Les plus anciennes traces d'écriture qui nous soient parvenues sont sur des tablettes d'argile qui ont tout l'aspect de livres de comptes. Les premiers idéogrammes chinois étaient destinés aux dieux : on les leur tendait sur des supports où il était attendu qu'ils répondissent en y inscrivant leurs propres marques sous la forme de fissures ou de craquelures par l'intermédiaire des rayons du soleil ou de tout autre agent naturel. Le besoin qui fut à l'origine des diverses écritures ne semble pas celui de fixer la langue. Destinées soit à établir des comptabilités, soit à rappeler et perpétuer les signes des dieux, elles renvoyaient au contraire à ce qui échappait aux fonctions du langage, elles étaient à l'écart de la littérature, elles demandaient encore à être traduites, elles instituaient des signes qui n'étaient pas des mots ou des mots appelés à rester hors des mots, paroles qui ne se souciaient pas de passer en bouche, imprononçables énigmes.

Il faut retenir de ses origines que l'écriture est un mode d'expression spécifique qui, profane ou sacré, ne peut être remplacé par aucun autre puisqu'il est né précisément de l'incapacité des autres à assumer sa fonction. Il faut retenir aussi que si elle est, comme la langue, les gestes ou le dessin, un moyen de communiquer, l'écriture s'inscrit dans l'absence, la solitude, le silence. Il 
faut retenir enfin que sa pratique confronte l'individu qui s'y livre à une matière par l'intermédiaire d'un instrument : en cela plus proche du feu surgi de la friction d'une pierre contre une autre, du dessin né sur le sable au moyen d'un bâton, sur la roche au moyen d'un caillou, elle suppose des stylets qui traceront sur l'argile ou la cire, des éclats de joncs imbibés de teinture pour laisser des lignes sur la surface d'un rouleau de papyrus, des burins qui graveront dans le marbre ou le cuivre, des gouges pour sculpter dans le bois, des plumes encrées pour tracer sur des parchemins, puis des craies, des crayons, des mines...

Il est presque étrange de noter combien les caractéristiques que s'est donnée l'écriture à ses origines sont restées dans la pratique que nous en avons communément aujourd hui et combien sont similaires les devoirs que nous attendons qu'elle remplisse, quand même ils ne sont pas explicites dans notre esprit. Il me semble évident que quiconque s'adonne à l'écriture ou entretient seulement la velléité de le faire aspire d'abord à se retirer du tourbillon des activités qui l'accaparent pour se livrer à cette vacance à même de le rendre disponible aux lubies secrètes, aux hantises inavouables, aux faiblesses dont il a honte, bref à ce qu'il sait être lui-même, dans sa finitude et ses malpropretés, dans ses manques et sa folie, que cela se traduise par une collection de timbres-poste, par l'ingestion d'un cassoulet, par les rêves d'un enfer ou l'appel à des visions. Nous ne retournons pas à la solitude, dont la pratique de l'écriture est la voie royale, sans chercher à retrouver ces atmosphères d'enfance dont, dans l'abandonnement poignant où il arrivait que nous fussions, dans le silence et l'immobilité de la chambre ou du recoin où il arrivait que nous soyons consignés, nous étions entourés de l'indifférence vertigineuse du monde, expérience terrible et en même temps saisissante qui nous livrait au vide incommensurable à l'entour de notre fragile pouls, à ce mystère de l'être, à l'énigme de sa raison et de son sens. Alors, déjà, sans le savoir, en venions-nous à écrire : du tranchant de nos semelles nous tracions dans la poussière, d'un éclat de bois nous griffions dans le plâtre d'un mur, d'un clou rouillé nous gravions dans un parquet...nous faisions des sottises.

Dans l'exercice de l'écriture il reste de cette pratique de nos inscriptions enfantines qui n'étaient pas faites pour dire explicitement quelque chose. Car qu'est-ce que l'écriture, à son origine comme aujourd'hui encore, que ce soit au moyen d'un crayon ou d'un clavier, sinon ces mêmes gestes, à peine endigués, à peine plus élaborés, de griffonnages, de grattages, de tapotements ? Ce sont aussi ces bruits infimes et propres à l'activité scripturaire, ce sont ces odeurs d'encre, de graphite, de ruban ou de poudre, ce sont ces sensations particulières suscitées par les natures diverses des feuilles de papier.

La posture et le geste qu'engendre l'écriture, à moins que, peut-être, ils ne l'aient engendrée eux-mêmes, comportent une dimension corporelle à la source du plaisir que l'on éprouve à s'y livrer, ou de la réticence qui en détourne. Il convient non seulement d'accepter cet assujettissement du dos, des bras, de la main et des doigts aux figures imposées par le dessin des lettres, 
par la forme des instruments et la nature des supports employés, mais encore de le rechercher et de le goûter, sachant que le plaisir proviendra autant du pétrissage de la langue et des surprises occasionnées par le brassage des concepts que des sensations issues de la rencontre entre la pointe de l'instrument et la surface qu'il entame. Des tournoiements commandés au poignet par la graphie jusqu'au centre de soi-même en passant par l'engrenage des muscles et des nerfs sollicités, c'est à de sensuelles acrobaties d'oiseau ou de nageur que le corps est livré, subissant des vrillements, des plongées, des piques, des virages, des rétablissements, de brusques ascensions, qu'on revienne sur le parcours effectué pour faire éclater une ligne, raturer ou surajouter, étendre de brusques espaces, répartir d'imprévus îlots ici et là, en segmenter ou en rapprocher d'autres, c'est à la jubilation de l'artiste entré en transes dans la composition d'une œuvre que l'on atteint, à cette danse que l'œuvre elle-même enfin devinée impose à son inventeur pour advenir. Il est une jouissance dans l'affrontement à la matière et à son façonnage rythmé dont est loin d'être privée l'écriture. Autant que toute discipline artistique, elle inscrit nos souffles, nos gestes, et tout ce qu'ils supposent, en amont, d'irréductiblement charnel, dans une énigmatique projection de notre corps.

Bien sûr il en va là seulement de la graphie mais il en ira d'une évidence plus flagrante encore dans ce qu'il sera convenu d'appeler le «style », qui n'est rien moins que la marque d'un corps unique sur un matériau commun, puisqu'avec l'évolution de l'écriture c'est la langue elle-même qui, au même titre que l'argile, la cire, le marbre ou le papyrus, est devenue « matière ». Depuis les premiers sumériens qui en tatouaient les statues de leurs divinités, depuis les premiers chinois qui tentaient par eux d'entrer en communication avec les puissances célestes, depuis les égyptiens qui leur confiaient la mission de transporter le récit de leurs vies au royaume des morts, tracer des signes est resté un moyen de tendre ce que l'on ne sait pas toujours de soi-même aux génies hypothétiques susceptibles de le révéler, de l'expliquer, et surtout de l'accueillir. C'est toujours, plus ou moins consciemment, chercher à provoquer un écho, et il se trouve en effet que, dans le cours même de cette activité, s'opèrent de mystérieuses césures qui composent un autre texte que celui que l'on projetait ou que l'on croyait écrire, en modifient le trajet, en transforment le sens. Rien n'est moins maîtrisable que ces signes et ces phrases qui, pour ne pas « s'envoler », se décomposent et se recomposent sans cesse autrement, comme si l'on ne faisait jamais qu'appliquer des grilles différentes sur le seul texte qui souhaite d'apparaître et qui, au gré des accidents, des résistances, des repentirs, de lettres mises à la place d'autres, de fautes de dyslexie, finit par se dessiner en filigrane, plus ou moins effacé et parcellaire, texte dont on n'atteint jamais l'inscription immédiate. Qu'est-ce que le sentiment d'avoir « un livre à écrire » sinon celui d'être porteur d'un texte déjà tout écrit mais dans une écriture et une langue si uniques, à ce point idiomatiques, que les matériaux dont on dispose ne sont pas plus habilités à les transcrire qu'ils ne le sont de nos songes quand nous nous y essayons à l'état de veille? 
Aussi bien n'est-ce jamais le texte que nous pouvons dire que nous voudrions écrire. Si la matière de l'écriture tend à se confondre avec la langue parlée et si l'écriture a pu devenir la littérature en se rendant capable d'inscrire les mythes, les légendes, les récits qui lui préexistaient, elle est restée un mode d'expression et de communication à l'écart de l'oralité. Les hommes l'ont chargée d'explorer en eux un champ non destiné à la divulgation orale, à l'échange, au dialogue, au théâtre, à l'éloquence. Dire et écrire sont deux modes de communication qui ne se recoupent pas.

Il n'est d'ailleurs que de constater les différences de forme et de contenu de nos propos selon que nous les tenons par oral ou par écrit. Le dialogue est toujours une partie subtile destinée à contenir la violence inhérente à toute rencontre et toujours à même de ressurgir du silence. Il est une condition de la vie sociale, la marque d'une communauté civilisée, policée. Il ne donne pas tant à connaître et à faire connaître qu'à rapprocher et à accorder, il suppose l'acceptation a priori d'un compromis. Son contenu dépend du niveau de fréquentation que les interlocuteurs ont déjà entre eux, des intentions qui les meuvent les uns à l'égard des autres, des enjeux de leur réunion, de leur disponibilité respective, mais aussi de l'image que chacun a de soi-même ou se découvre sous le regard des uns et des autres. La conversation est un exercice de langage contrôlé dans lequel il s'agit de prendre le moins de risques possible et au cours duquel l'emprise du langage est précisément la plus forte parce que le débit exige, la répartie inspirée, la réponse attendue ne permettent que de dire du déjà dit ou ce que la logique et les particularités du langage le disposent à formuler de lui-même. On y brille à proportion de sa transparence à la langue, c'est-à-dire à proportion de la facilité de ses facultés à la reproduire. Il est possible de s'en suffire et même de s'y accomplir. On ne manque pas, toutefois, d'en sortir avec la sensation de n'avoir rien dit, ou trop peu, ou d'avoir dévié et exprimé autre chose que ce que l'on avait eu l'intention de formuler, en somme d'avoir parlé à côté de soi. On en revient toujours aussi lourd d'un non-dit qui sans doute n'était pas exprimable puisque les mots ont été et restent incapables de s'en saisir.

L'écriture ne présente pas moins de limites que le langage mais elle exprime au moins que la nature du désir de dire comporte le désir de dire même ce qui n'est pas dicible, ce qui n'est traduisible en aucune langue, ce qui est à proprement parler ineffable. Elle tend à détourner le langage de sa fonction sociale et, en ce sens, à le retourner contre lui-même dans la mesure où elle conteste sa prétention à traduire notre individualité, ou elle tâche à l'arrêter, à le raturer, à le déformer pour que s'y inscrive, fût-ce sous traces de fautes, d'écarts, de blessures, de balafres et de paraphes, autre chose que le sens commun. Elle veut au moins contrôler son débit et son rythme, faire traduire aux mots le mouvement d'un regard, la mesure d'une respiration, la particularité d'une sensibilité, le pouls d'une existence singulière. A écrire nous aspirons et travaillons à nous donner aussi intégralement que possible à toucher. 


\section{IV}

Il convient de noter que, pour chercher à traduire leur individualité irréductible, les hommes et les femmes qui écrivent sont emportés dans des courants qui les mêlent et en viennent à traiter de sujets qui se confondent. Ce que, des plus anciennes formes littéraires comme l'épopée ou le poème jusqu'aux plus récentes comme le roman ou l'autobiographie, explorent les ouvrages se réduit à un nombre très restreint de thèmes récurrents. Depuis ses origines la littérature n'a guère pour auteur que « l'éternel humain ».

L'enfance, sur laquelle il est rare en vérité qu'aucun ouvrage ne revienne de façon explicite, suffit souvent à être en elle-même l'objet et la matière comme naturels de nombre de livres, elle paraît apte à justifier à elle seule qu'on ait entrepris de les écrire, elle va jusqu'à sembler donner pour rôle unique à l'écriture de la retrouver, de la reposséder, de remonter aux sources de ce que l'on est, d'y découvrir l'explication de tout ce que l'on a fait. Heureuse ou malheureuse, ressurgissant dans un élan de nostalgie ou de vengeance, elle témoigne des possibles qui n'ont pas été réalisés, des promesses qui n'ont pas été tenues, des dons qui ont été gâchés ou dont la vie s'est repue en n'en laissant rien à celui qu'elle en avait doté. Les mémoires, les confessions bien sûr sont sous-tendus par un tel sentiment mais aussi les ouvrages historiques, voire sociologiques, qui reposent sur le désir plus ou moins conscient de se situer dans une lignée, de se donner une légitimité, car il est bien difficile de trouver en soi-même sa raison d'être et la fonction de sa présence au monde. Et l'enfance, la nôtre comme celle de l'humanité, représente un état de candeur sinon perdu du moins atrophié : c'était l'époque où il semble que nous étions au plus près de l'Être parce que la sensibilité non voilée par le langage y était à l'apogée de son acuité.

Encore que tout souvenir d'enfance en soit un, il faut sans doute distinguer les ouvrages engendrés par le travail de deuil, qui débutent et reposent sur la perte d'un être cher : père, mère, amant, épouse, etc. Toute séparation arrache si violemment une part de soi, confronte si brutalement au vide qu'il faut colmater cette béance dèmesurée, occuper la disponibilité hébétée qu'elle procure, combler le manque, pour retrouver quelque autonomie. Le cri ne suffit pas à exprimer ni à chasser la souffrance alors constitutive, permanente, engendrée par le coup reçu. L'écriture contrebalance un vertige qui menace de précipiter dans le néant. Quand même elle remue le couteau dans la plaie en donnant sur l'objet perdu un regard plus aigu, en exaltant ses qualités, en soulignant son originalité irremplaçable, elle permet en somme d'intérioriser ce que la présence, paradoxalement, soustrayait dans l'hermétique clôture de l'enveloppe de peau et par l'indépendance de l'autre.

L'écriture est une thérapie, elle a fonction aussi de catharsis : il est courant d'en user pour se purifier des monstruosités, des contrariétés qui, aigrissant en encombrant tout l'être, finissent par le menacer de folie ou de mort. Elle permet de circonscrire l'origine du mal si celui-ci est précis : une obsession, une 
insatisfaction, une culpabilité, l'isolement dû à une disgrâce, à une damnation injuste, à l'accablement du sort, à un handicap plus diffus. La condition humaine se caractérise par la souffrance parce que l'homme est en perpétuelle contradiction avec son désir, dont il ne connaît pas même clairement l'objet. Aimer est un idéal jamais atteint, et être aimé est gratifiant sans doute mais ce ne l'est jamais non plus de la manière dont on voudrait l'être. Cette souffrance est un état qui se traduit de façon insidieuse : malaise que l'on traîne avec soi telle cette sensation agaçante née de l'oubli d'une action à faire ou d'un objet à prendre et qu'on ne parvient pas à identifier, ou bien prurit exaspérant, colère qui rend toute chose agressive, mécontentement sans cause qui fait voir tout en noir. Ecrire est d'abord - et souvent uniquement - exprimer cette contrariété. Ce peut être tenter d'en cerner la raison, cette raison n'étant jamais déterminante. C'est décrire l'être humain étouffant dans ses entraves, retenu par les liens que constituent ses limites, empêché par ses manques.

L'écriture témoigne enfin d'une violence à s'imposer qui dépasse les contenus dont on la charge. Serait-il possible d'écrire seulement pour soi ou pour le plaisir de manier le langage par l'écriture, pour les conditions que suppose son acte, pour l'état dans lequel il plonge par rapport à la trivialité de l'existence ? Écrire n'est guère dissociable de l'idée, même inavouée ou latente, de laisser une trace qui perdure au-delà de soi et plus longtemps. C'est là un désir qui se confond avec la nécessité pour tout être vivant de s'opposer aux forces d'anéantissement, de se dresser face aux autres, de valoir au regard des montagnes et du ciel. C'est là également une réaction à l'insatisfaction existentielle dont on en vient à attribuer la responsabilité aux principes erronés qui gouvernent les hommes. L'ambition de refaire le monde ou tout au moins de le réformer est l'envers de l'aspiration à plus de bonheur pour soi, quitte à s'acculer à soutenir qu'on veut changer la vie tout en entrevoyant que son intérêt serait le statu quo : que le monde fût autre ne supprimerait-il pas la raison d'être que l'on se trouve dans l'écriture?

Celle-ci demeure primordiale, quelque justification qu'on lui donne, parce qu'elle répond à ce désir de dire qui taraude alors même que l'on n'a rien à dire ou qu'on ignore ce que l'on a l'impression de vouloir dire. Des auteurs récents assument cette conception la plus nue, la plus pauvre mais aussi la plus fondamentale, peut-être, de l'écriture. Hors des médiations de la fiction, des sublimations de la poésie ou des ressorts de la littérature didactique, ils se bornent à l'urgence de se dire, pratique pathétique où l'écriture consomme tellement du temps de la vie que la vie même finit par ne pouvoir plus se nourrir que de l'acte d'écrire, ou l'écriture et la vie entrent en une telle concurrence dans la course à la mort, comme le cas d'Hervé Guibert en a montré l'évidence, qu'il n'est plus question que de vivre pour écrire et d'écrire pour vivre. L'écriture n'est guère qu'un prétexte pour se soumettre à l'urgence de cet élan qui, en nous, exige d'être aussitôt apporté, et qui, inéluctablement, prend celui qui écrit en otage, corps, souffle et langue. 


\section{v}

Les écrivains que les siècles ont retenus et ceux auxquels le notre a conféré assez de notoriété pour laisser envisager qu'ils subsisteront dans les siècles futurs, encore que ces consécrations ne s'assortissent d'aucune garantie qui puisse les rendre définitives, illustrent plus ou moins les quelques mobiles que l'on peut voir à l'écriture. En ce sens, ils ne se distinguent pas de tous ceux qui, s'étant retirés pour écrire, n'ont cependant point vu leur ouvrage édité ou recevoir un accueil propre à les sortir de l'anonymat. Ils ont suivi le trajet de quiconque éprouve l'écriture comme l'idéal auquel son être tend, ce que l'on peut éprouver fort tôt, à un âge oủ non seulement il parait difficile d'acquérir les techniques aptes à rendre maître de cet instrument, mais où l'expérience et la vie mêmes ne sauraient avoir fourni déjà le matériau nécessaire à l'expression de quoi que ce soit. Si la précocité d'un Rimbaud prouve que le désir de dire surgit très tôt, le contenu de ses poésies anticipe de beaucoup ce qu'il a jamais vécu et n'est pas en soi des plus original. Il a donc fallu à nos écrivains attendre que se constituent en eux une matière sous l'apparence de souvenirs, de blessures, d'images, avant de pouvoir lui donner forme en lui trouvant un sens. Le besoin d'être acceptés dans leur singularité soutenait leur énergie, accompagné peut-être de l'intention moins pure de provoquer, mettre à l'épreuve, tester la validité des sentiments d'autrui à leur égard, déranger. Par son objet autant que par sa nature, l'écriture ne se départit pas d'une dimension subversive. Ce que l'écrivain apporte s'oppose à la norme qui ne saurait être pour lui qu'apparence et mensonge. Ce qu'il exprime sans forcément le dire mais par le seul fait d'écrire est un scandale parce que toute authenticité tranche sur l'admissible et le convenu. Les écrivains reviennent eux aussi sur ces moments où nous prenons mesure de l'existence que nous font notre hérédité, notre éducation, nos choix, eux-mêmes tributaires de nos limites. Ils travaillent la violence et l'anxiété que nous vivons tous jusqu'a la nausée face au désir, et face à l'amour même qui nous est porté. Ils écrivent pour surmonter le dégoût dont nous ne guérissons jamais tout à fait et que nous inspirent notre besoin de l'autre et la vision aiguë que ce besoin donne des corps. Ils écrivent dans la perspective d'une réhabilitation à leurs propres yeux de leur vécu, pour le choisir a posteriori en quelque sorte, pour le célébrer en somme, mais toujours avec cette intention sous-jacente de remettre en cause les explications, de contester les chansons.

De page en page, de livre en livre, les écrivains, et c'est en cela qu'ils se démarquent sans doute, prennent mesure des pouvoirs de l'écriture. Celle-ci n'est pas tant un outil pour publier ce qui a été vécu qu'un art de déployer des impressions, des perceptions, des sentiments, des intuitions qui, dans la fulgurance de l'expérience, ont été trop vite brûlés, elle amène à découvrir leur étendue et leur profondeur, elle n'en livre pas la signification mais en fait la surprise. Dès lors elle permet de passer du monde des idées et des valeurs dans lequel nous sommes plongés mais auquel nous n'appartenons pas dans un 
univers personnel et intime, à savoir un monde si individuel et irréductible qu'au regard de la société il ne peut guère paraître que fictif, loufoque, scabreux, et tout simplement incompréhensible. Bien sûr, la dialectique culturelle, les idées dans l'air du temps, les influences dont nous sommes tissés, et ne serait-ce que la syntaxe et le vocabulaire réinsèrent dans des schémas de réflexion et de représentation qui retirent l'écriture des fissures qu'elle tentait d'agrandir, lui ôtent de sa faculté exploratrice, voire destructrice. Le miracle est que l'œuvre composée avec cette force-là fasse écho chez les lecteurs, se voie « récupérée », échoue, en définitive, à proportion du succès qu'elle rencontre. Des écrivains n'en ont pas été dupes qui se sont attachés à renier leurs écrits ou à en contester les interprétations, à accentuer leur particularité jusqu'au point de désarçonner, ont choisi la folie, comme s'ils n'avaient eu de cesse de se dégager des mouches toujours trop promptes à se jeter en masse sur leurs productions.

Il y a dans l'écriture un ressort qui l'éloigne de l'enregistrement sans fin des apparences successives, et des modes, pour inventer des cohérences nouvelles, explorer des possibles, imposer des évidences qui n'existent pas. Si une œuvre était destinée à ne dire que la vie telle qu'elle est, dans le cadre social tel qu'il est, avec ses valeurs et ses routines, elle n'aurait pas grand intérêt. Si le talent ne consistait qu'à exprimer un peu mieux que tout le monde ce que pense tout le monde, il ne ferait qu'étrécir le champ de l'écriture et de la langue. Le plaisir qu'on éprouverait à lire serait alors de l'ordre du narcissisme et de la complaisance, il ne serait pas le symptôme d'un approfondissement des facultés intellectuelles et d'un écarquillement des sens.

Il en va là, sans doute, de la plus grande part de ce qui se publie et se lit, et c'est une des raisons pour lesquelles si peu d'œuvres surnagent à la surface des siècles. Il n'est pas d'œuvre qui reste et qui compte dont l'auteur n'ait écrit en retournant les mots contre ce qu'il était convenu de leur faire dire, non qu'il se soit agi explicitement pour lui d'obéir à un désir de licence en butte à l'ordre établi, mais parce qu'il éprouvait une exigence de présence. Son écriture revendiquait la jubilation que tout être à vocation de connaittre par son corps, par son intelligence, dans le monde concret et tangible auquel il appartient et qui, d'une certaine manière, ne cesse de l'attendre.

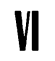

Le désir de dire ne suffit pas à bâtir une œuvre parce que ce qui en est à l'origine n'est le propre de personne et parce que « dire » n'est pas nécessairement l'articulation d'une parole. L'écriture est un artisanat qui use de quelques instruments, d'un petit nombre de supports, et d'une matière : la langue. Elle accède à la littérature quand on ne l'emploie plus pour échanger des informations, pour communiquer, pour traduire, mais pour dire ce qui ne se dit pas. Elle devient créatrice quand elle subvertit les médiations par lesquelles il lui faut passer, qu'elles relèvent du langage, des genres littéraires, des symboles, des formes. Ce sont là les maigres et peu fiables critères qui permettent de 
parier que l'on a affaire à une œuvre authentique, à un ouvrage préfabriqué ou illusoirement original. Qu'il y ait des auteurs à respecter n'entraine pas qu'il y ait des lois auxquelles il faudrait se soumettre, bien au contraire, mais un ouvrage ne s'impose comme tel s'il ne présente pas une cohérence interne, c'est-a-dire si nulle nécessité ne le structure.

Aucune définition de la littérature ne dispensera le lecteur, hélas, de lire le tout-venant de la production littéraire, quitte à perdre de son précieux temps à des ouvrages qui ne lui laisseront rien. Il est vrai que c'est là l'ambition affichée de bien des littérateurs, lesquels ne voient d'autre mission aux livres que de faire passer le temps, c'est-à-dire de faire oublier que le temps passe. Sera-t-il permis, nonobstant, de regretter que, faute de considérer les propriétés spécifiques de l'écriture, on promeuve une littérature pasteurisée ou convenue qui cherche plutôt à s'en débarrasser, qui veuille gommer comme les marques d'une origine honteuse toute trace d'application, tout rappel de bruits de plume sur le papier, tout ordonnancement des mots qui s'écarterait du langage parlé réduit au plus petit dénominateur commun, toute manifestation de présence, toute opacité, toute résistance, toute musique, toute couleur, toute saveur... ? Et pourtant, si l'on y prend garde, qu'est-ce qui sauve les œuvres du passé et donne à la littérature un statut de médiation irremplaçable sinon ce qui constitue sa matière : l'écriture, la forme unique que chacun est susceptible de lui donner, la nature concrète, presque charnelle, qu'elle présente, les irisations que sa linéarité suscite dans les mots, la dimension spatiale atteinte par le contenu même du fait de son étalement, de sa répartition, de ses symétries obtenues par son inscription... ? C'est peu de lire si, en lisant, la possibilité ne nous est pas offerte de nous hisser jusqu'au cœur de l'artiste à l'ouvrage pour y percevoir avec lui les rythmes, les vertiges, les risques, les terreurs, les surprises et les grâces de l'œuvre que l'écriture engendre. 